\title{
Imipenem-resistant Pseudomonas aeruginosa producing IMP-1 metallo- $\beta$-lactamases and lacking the outer-membrane protein OprD
}

Pseudomonas aeruginosa is a common nosocomial pathogen, particularly among immunocompromised patients.

Carbapenems, mainly imipenem and meropenem, are useful agents for the treatment of infections due to multi-resistant pseudomonads because these drugs are even stable against extended-spectrum and AmpC- $\beta$ lactamases (Livermore, 1995). However, the imipenem resistance rate of $P$. aeruginosa has risen in our laboratory. Production of metallo- $\beta$-lactamases (MBLs) and deficiency of the outer-membrane protein OprD are basic mechanisms of resistance to imipenem in $P$. aeruginosa. MBLs hydrolyse virtually all $\beta$-lactams and are insensitive to clinically available inhibitors. IMP-1 was the first MBL described in $P$. aeruginosa (Watanabe et al., 1991), but subsequently IMP-type MBLs have been detected in other countries of the Far East, as well as in Europe, Canada and Brazil, indicating a wide distribution of these resistance determinants. To date, 21 types of blaIMP have been published in GenBank (http://www.lahey.org/studies/other.asp). The MBL genes are horizontally transferable because they are inserted in integrons, and some of these integrons are located on conjugative plasmids (Poirel et al., 2000). Due to its ability to spread, carbapenem resistance related to MBL production has become a serious concern (Richet et al., 2001).

In 2004, we reported seven carbapenemresistant $P$. aeruginosa isolates producing IMP-1 MBLs at a university hospital in China (Wang \& Mi, 2004). We now report on other factors that codetermine imipenem resistance in P. aeruginosa 68549.

$P$. aeruginosa 68549 was isolated in January 2002 from an inpatient with a pulmonary infection, and was identified by Vitek Gram-negative identification cards (bioMérieux). One strain of $P$. aeruginosa carrying the $b l a_{\mathrm{IMP}-1}$ gene was used as a positive control for the detection of the $b l a_{\mathrm{IMP}}$ gene. $P$. aeruginosa ATCC27853 was used as a negative control, and for quality control of the antimicrobial-susceptibility test. Antimicrobial-susceptibility tests for MBL-producing $P$. aeruginosa were performed by the microdilution method with cation-adjusted Mueller-Hinton broth (Oxoid) according to the recommendations of the National Committee for Clinical Laboratory Standards (2004). The MICs of potent antibiotics for $P$. aeruginosa, including piperacillin, ceftazidime, cefotaxime, cefazolin, cefalotin, cefoxitin, aztreonam, imipenem, gentamicin and ciprofloxacin (Oxoid), were determined. A disc diffusion test, with 2-mercaptopropionic acid as an MBL inhibitor, was used to screen for MBL producers, as described by Arakawa et al. (2000).

Template DNA for PCR was prepared by a rapid alkaline lysis procedure (Takahashi \& Nagano, 1984). The following primers for $b l a_{\mathrm{IMP}}$-specific amplification were used: 5'-CGG CC G/T, CAG GAG A/C G G/T, CTT, T- $3^{\prime}$ and $5^{\prime}$-AAC CAG TTT TGC C/T TT AC C/T AT-3', designed based on all IMP-type MBLs published in GenBank; 5'-GCG CAT CTC CAA GAC CAT G-3' and $5^{\prime}$-GCC ACG CGA TTT GAC GGA G-3', for amplification of the outer-membrane protein OprD-encoding gene. PCR conditions for both kinds of genes were $3 \mathrm{~min}$ at $93^{\circ} \mathrm{C}, 40$ cycles of $1 \mathrm{~min}$ at $93^{\circ} \mathrm{C}, 1 \mathrm{~min}$ at $55^{\circ} \mathrm{C}$ and $1 \mathrm{~min}$ at $72^{\circ} \mathrm{C}$, and finally, $7 \mathrm{~min}$ at $72^{\circ} \mathrm{C}$. The amplicons were purified with PCR Clean up kits (Roche Molecular Biochemicals) and were sequenced on an ABI PRISM377 sequencer analyser (Applied Biosystems).

$P$. aeruginosa 68549 was highly resistant to imipenem and to other $\beta$-lactams except aztreonam and ciprofloxacin. The isolate was indicated as a producer of MBLs using the disc diffusion test, and was positive in PCR assays with $b l a_{\mathrm{IMP}}$-specific oligonucleotide primers, where a product of 587 bp was obtained. The aligned sequence was analysed with BLAST 2.0 and found to be identical to blaIMP-1 from Serratia marcescens (GenBank accession no. S71932), $P$. aeruginosa (GenBank accession no AJ223604) and Klebsiella pneumoniae (GenBank accession no D29636). The patient had no history of recent travel to Japan and Singapore. Undetected importation by other patients or travellers is possible.

P. aeruginosa 68549 was negative with OprD gene-specific oligonucleotide primers, which showed that the isolate lacked the outer-membrane protein OprD compared with $P$. aeruginosa ATCC27853 and with imipenem-susceptible $P$. aeruginosa controls. It seems likely, from its mass, that the OprD corresponds to a major porin and that high-level resistance to carbapenems requires impermeability as well as an IMP $\beta$-lactamase. Microbiologists should be aware that Gram-negative bacteria with borderline susceptibility to carbapenems could be IMP producers. Suspicious isolates should have their carbapenem MICs checked and should be examined for carbapenemase activity.

\section{Acknowledgements}

We are grateful to Yun-Song Yu, Department of Infectious Diseases, No. 1 Affiliated Hospital, College of Medicine, Zhejiang University, Hangzhou, China, for his generous provision of the $b l a_{\mathrm{IMP}}$-positive control strain and technical assistance.

\section{Chun-Xin Wang ${ }^{1}$ and Zu-Huang $\mathrm{Mi}^{\mathbf{2}}$ \\ ${ }^{1}$ Clinical Laboratory, Wuxi No.1 Hospital, Nanjing Medical University, Wuxi, China \\ ${ }^{2}$ Wuxi Clone Gen-Tech Institute, Wuxi, China}

Correspondence: Chun-Xin Wang (bacteria001@163.com) 
Arakawa, Y., Shibata, N., Shibayama, K., Kurokawa, H., Yagi, T., Fujiwara, H. \& Goto, M. (2000). Convenient test for screening metallo- $\beta$-lactamase-producing gram-negative bacteria by using thio compounds. J Clin Microbiol 38, 40-43.

Livermore, D. M. (1995). $\beta$-lactamases in laboratory and clinical resistance. Clin Microbiol Rev 8, 557-584

National Committee for Clinical Laboratory Standards (2004). Performance Standards for Antimicrobial Susceptibility Testing. Fourteenth informational supplement. NCCLS document M100-S14, vol. 24, no. 1, pp. 36-38. Wayne, PA: NCCLS.
Poirel, L., Naas, T., Nicolas, D., Collet, L., Bellais, S., Cavallo, J. D. \& Nordmann, P. (2000). Characterization of VIM-2, a carbapenem-hydrolyzing metallo-beta-lactamase and its plasmid- and integron-borne gene from a Pseudomonas aeruginosa clinical isolate in France. Antimicrob Agents Chemother 44, 891-897.

\section{Richet, H. M., Mohammed, J.}

McDonald, L. C. \& Jarvis, W. R. (2001).

Building communication networks: international network for the study and prevention of emerging antimicrobial resistance. Emerg Infect Dis 7, 319-322.
Takahashi, S. \& Nagano, Y. (1984). Rapid procedure for isolation of plasmid DNA and application to epidemiological analysis. J Clin Microbiol 20, 608-613.

Wang, C.-X. \& Mi, Z.-H. (2004). IMP-1 metallo-beta-lactamase-producing Pseudomonas aeruginosa in a university hospital in the People's Republic of China. J Antimicrob Chemother 54, 1159-1160.

Watanabe, M., lyobe, S., Inoue, M. \& Mitsuhashi, S. (1991). Transferable imipenem resistance in Pseudomonas aeruginosa. Antimicrob Agents Chemother 35, 147-151. 